proliferation of VSMCs and play a functional role in atheroprotection. Efforts aiming at enhancing oestrogen receptor expression and/or activity may prove to be an attractive alternative therapy against atherosclerosis.

\section{e0106 THE EFFECT OF ADENOSINE AND ISCHAEMIA POSTCONDITIONING ON MMP-2 AND MMP-9 IN RABBIT ISCHAEMIA REPERFUSION MYOCARDIAL}

doi:10.1136/hrt.2010.208967.106

${ }^{1}$ HeXiaonan, ${ }^{2} Y u$ Chen, ${ }^{3}$ Li Shumei. ${ }^{1}$ Department of Cardiology, TianJin Chest Hospital, TianJin; ' ${ }^{2}$ epartment of Cardiology, China-Japan Union Hospital, Jilin University, Changchun; ${ }^{3}$ Department of Cardiology, the Second Hospital of Jili University, Changchun

Objective To observe the effect of adenosine and ischaemia postconditioning on MMP-2 and MMP-9 in rabbit ischaemia reperfusion myocardial.

Methods The rabbits were divided into four groups in basic experiment: control group, antagon group, postconditiong group and adenosine group. The activity of MMP-2 and MMP-9 was observed by gelatin zymography and the expression of MMP-2 and MMP-9 was observed by RT-PCR and Western Blot.

Results The results of RT-PCR showed that the light density of MMP-2/GAPDH $(0.76 \pm 0.22)$ in adenosine group and postconditioning group was slightly lower than that of control group and antagon group $0.80 \pm 0.20$ ( $p>0.05)$. The light density of MMP$2 / \mathrm{GAPDH}(0.77 \pm 0.12)$ in adenosine group and postconditioning group was significanty lower than that of control group and antagon group $1.30 \pm 0.10(p<0.01)$. There was no dramitic difference between adenosine group and postconditioning group. The results of Western blot showed that MMP-9 and MMP-2 in adenosine group and postconditioning group was lower than control group and antagon group. The results of Zymography revealed that the light density of MMP-9 in adenosine group and postconditioning group much lower than those in control group and antagon group $(p<0.05)$. There was no difference between adenosine group and postconditioning ( $p>0.05)$.

Conclusions Adenosine and postconditioning can decrease the expression and the activity of MMP-9 and inhibit the inflammation, relieving the ischaemia reperfusion injury.

\section{e0107 CARDIAL PROTECTIVE EFFECTS OF DIFFERENT DOSAGE ATORVASTATIN IN PATIENTS WITH STABLE ANGINA AFTER PERCUTANEOUS CORONARY INTERVENTION}

doi:10.1136/hrt.2010.208967.107

${ }^{1}$ Jin-Zi Su, ${ }^{2}$ Guang-Ling Chen, ${ }^{1}$ Wen-Qin Cai. 'Department of Cardiology, First Affiliated Hospital, Fujian Medical University, Fuzhou, ${ }^{2}$ Ming Dong Hospital, Fujian

Background The incidence of myocardial injury limits the clinical outcomes of percutaneous coronary intervention (PCI). This randomised controlled study was designed to evaluate the protective effects of pretreatment atorvastatin on myocardial injury and inflammatory reaction after PCI.

Methods 82 patients with chronic stable angina without previous statins treatment in 2 months before PCI were randomised to receive atorvastatin $10 \mathrm{mg} / \mathrm{qn}$ (group $\mathrm{A}, \mathrm{n}=27$ ), $20 \mathrm{mg} / \mathrm{qn}$ (group $\mathrm{B}$, $\mathrm{n}=28$ ) or $40 \mathrm{mg} / \mathrm{qn}$ (group $\mathrm{C}, \mathrm{n}=27$ ) treatment for 3 days before PCI. CK-MB, cTnI, hsCRP, IL-6, sICAM-1 were measured at baseline, 8 and $24 \mathrm{~h}$ after the procedure. 1-month clinical follow-up was obtained by office visit in all patients.

Results The peak levels of CK-MB and cTnI were increased significantly in all three groups $24 \mathrm{~h}$ after PCI (all $\mathrm{p}<0.05)$. Either elevation above the upper normal limit (UNL) or $>3 \times$ UNL of $c T n I$, there were significant differences between group $A$ and $B(p<0.05)$, and between group $A$ and $C(p<0.05)$, but no difference between group $B$ and $C$ $(p>0.05)$. Similarly changes were also found in CK-MB. The level of IL-6, sICAM-1 and hsCRP $8 \mathrm{~h}$ after PCI were higher than those before PCI (all $p<0.05)$. There were significant differences in IL- 6 and hsCRP among the three groups (all $\mathrm{p}<0.05$ ), but no significant difference in sICAM-1 ( $p>0.05)$. The level of hsCRP and sICAM-1 $24 \mathrm{~h}$ after PCI were higher than those $8 \mathrm{~h}$ after PCI in all three groups (all $p<0.05)$, but IL- 6 significantly decreased $(p<0.05)$. There were significant differences among the three groups (all $\mathrm{p}<0.05$ ). No serious cardiovascular events occurred during follow-up.

Conclusion Even short term pretreatment with atorvastatin before PCI may reduce procedural myocardial injury by reducing inflammatory factors in chronic stable angina patients.

\section{Q0108 ESTROGEN INDUCES RECOVERY OF INJURED ARTERY ENDOTHELIUM BY MOBILISING ENDOTHELIAL PROGENITOR CELL}

doi:10.1136/hrt.2010.208967.108

Xiaohui Zhao, Yangguang Yin, Yuqiang Fang, Jianfei Chen, Bin cui, Lan Huang. Cardiovascular Disease Research Center, Xinqiao Hospital, Third Military Medical University, Chongqing, China

Introduction Mobilization of endothelial progenitor cells (EPC) restores endothelial function, representing a novel therapeutic direction for injured blood vessel recovery. The present study was designed to determine the effect of oestrogen on EPC mobilisation and regeneration of endothelium in mice.

Methods Variectomy is performed before treatment of $17 \beta$-oestradiol, 17 $\beta$-oestradiol combination with oestrogen receptor agonistICI182780 and 17 $\beta$-oestradiol with PI3K blockers- LY294002. Then, carotid artery injury was preformed and neointima was evaluated by H.E staining. 1 and 3 days later, mobilisation of EPCs was evaluated by FACS as double positive of Sca-1/VEGFR-2. Evens blue was injected and area of reendothelization was calculated after 7 days. To trace EPCs in vivo, $1 \times 10^{6}$ autologous spleen-derived EPCs were labelled with DAPI and transplanted through tail vein.

Results 1 or 3 days after carotid artery injury, EPCs of peripheral blood were $0.42 \pm 0.135 \% \quad(n=6), 1.47 \pm 0.38 \%(n=5) \quad$ (ovariectomy $+E 2)$ $0.13 \pm 0.024 \%(n=6), 0.25 \pm 0.024 \%(n=6)$ (ovariectomy); $0.43 \pm 0.16 \%$ $(\mathrm{n}=6), 0.65 \pm 0.21 \%(\mathrm{n}=4)$ (non-ovariectomy); $0.12 \pm 0.019 \% \quad(\mathrm{n}=6)$, $0.25 \pm 0.062 \%(n=6)$ (ovariectomy $+E 2+L Y)$ and $0.12 \pm 0.019 \%(n=6)$, $0.24 \pm 0.067 \%(\mathrm{n}=6)$ (ovariectomy $+\mathrm{E} 2+\mathrm{ICI})$. Area of re-endothelization were (ovariectomy, $28.33 \pm 13.49 \%, \mathrm{n}=5$ ) vs (ovariectomy $+\mathrm{E} 2$, $69.53 \pm 14.14 \%, \mathrm{n}=5$ ) vs (non-ovariectomy, $83.11 \pm 7.94 \%, \mathrm{n}=4$ ) $(\mathrm{p}<0.01)$. In vivo tracing experiment detected blue fluorescence cells in injured sites that were also positive of CD31, indicating EPCs homing to target sites.

Conclusion Ooestrogen can induce EPCs mobilisation through ERs/ PI3K pathway which is helpful to promote endothelium recovery of injured carotid artery.

\section{e0109 THE EFFECTS OF C-REACTIVE PROTEIN ON TOLL-LIKE RECEPTOR 4 SIGNAL TRANSDUCTION ON CD14+ MONOCYTE}

doi:10.1136/hrt.2010.208967.109

Jinlai Liu, Long Peng, Yanting Luo. Department of Cardiology, The Third Affiliated Hospital, Sun Yat-sen University, Guangzhou, China

Objective To observe the effects of C-reactive protein (CRP) on Tolllike receptor 4 (TLR4) expression in $\mathrm{CD} 14^{+}$monocyte in human, and the role of CRP in the inflammatory mechanism.

Methods $\mathrm{CD}_{14}{ }^{+}$monocytes were isolated from blood in healthy volunteers by the Ficoll density gradient and stimulated by CRP 